\title{
A Case of Idiopathic Systemic Capillary Leak Syndrome with High Serum Levels of G-CSF on Exacerbation
}

\author{
Naoki Nakagawa ${ }^{1}$, Hisanobu Ota ${ }^{1}$, Yasuko Tanabe ${ }^{1}$, Maki Kabara ${ }^{1}$, Motoki Matsuki ${ }^{1}$, \\ Junko Chinda $^{1}$, Naka Sakamoto ${ }^{1}$, Takayuki Fujino ${ }^{1}$, Naofumi Takehara ${ }^{2}$, \\ Toshiharu Takeuchi ${ }^{1}$, Jun-ichi Kawabe ${ }^{2}$, Nobuyuki Sato ${ }^{1}$, Yuichiro Kawamura ${ }^{1}$, \\ Takashi Fukuhara ${ }^{3}$, Katsuya Ikuta ${ }^{4}$, Kenjiro Kikuchi $^{5}$ and Naoyuki Hasebe ${ }^{1}$
}

\begin{abstract}
Systemic capillary leak syndrome (SCLS) is a life-threatening disorder which presents with periodic episodes of hypovolemic shock, due to plasma leakage to the extra-vascular space reflected by accompanying hypoalbuminemia, hemoconcentration and edema often with associated monoclonal gammopathy. We describe a 28-year-old woman with SCLS who required aggressive fluid resuscitation and was successfully treated with corticosteroid, terbutaline, and theophylline. At exacerbation, the levels of serum granulocyte colony-stimulating factor (G-CSF) were increased. Thus, G-CSF might play an important role and can be a useful biomarker for the severity of attacks in SCLS.
\end{abstract}

Key words: systemic capillary leak syndrome, granulocyte colony-stimulating factor

(Intern Med 50: 597-600, 2011)

(DOI: 10.2169/internalmedicine.50.4857)

\section{Introduction}

Systemic capillary leak syndrome (SCLS) is a very rare idiopathic disorder which presents with periodic episode of hypovolemic shock, due to plasma leakage to the extravascular space reflected by accompanying hypoalbuminemia, hemoconcentration and edema, and in most cases, the presence of a paraproteinemia $(1,2)$. Clinical signs during attacks result from extravasation of large volumes of plasma from the intravascular space to the interstitial space. The secondary development of compartment syndrome or rhabdomyolysis from the swelling of muscle compartments is a rare complication. Clarkson described the first case of SCLS in 1960 (1), and about 150 patients have been described since (2-6). The frequency of reports has increased in the past decade, probably due to an increased clinical awareness of the syndrome, however the true incidence of SCLS may be underestimated. Clinicians need to be aware that this syndrome has an underlying diagnostic difficulty and carries a high mortality. Thus, it is hoped to discover a new molecule to monitor this syndrome. We present a patient with SCLS who was treated successfully with corticosteroid, beta-2 agonists and theophylline and found a relationship between serum levels of granulocyte colony-stimulating factor and the severity of attacks in SCLS.

\section{Case Report}

A 28-year-old woman was admitted to our hospital with generalized edema and circulatory shock in July 2006. She had previously been hospitalized three times at another hospital over the past 6 months with oliguria, hypotension and generalized edema. She presented at the emergency room with oliguria and a feeling of dizziness and fatigue. On examination her systolic blood pressure was $82 \mathrm{mmHg}$, pulse

\footnotetext{
${ }^{1}$ Division of Cardiology, Nephrology, Pulmonology and Neurology, Department of Internal Medicine, Asahikawa Medical University, Japan, ${ }^{2}$ Department of Cardiovascular Regeneration and Innovation, Asahikawa Medical University, Japan, ${ }^{3}$ Depatment of Internal Medicine, Asahikawa City Hospital, Japan, ${ }^{4}$ Division of Gastroenterology and Hematology/Oncology, Department of Internal Medicine, Asahikawa Medical University, Japan and ${ }^{5}$ Department of Cardiology, Hokkaido Junkanki Hospital, Japan

Received for publication November 15, 2010; Accepted for publication December 20, 2010

Correspondence to Dr. Naoki Nakagawa, naka-nao@asahikawa-med.ac.jp
} 
A

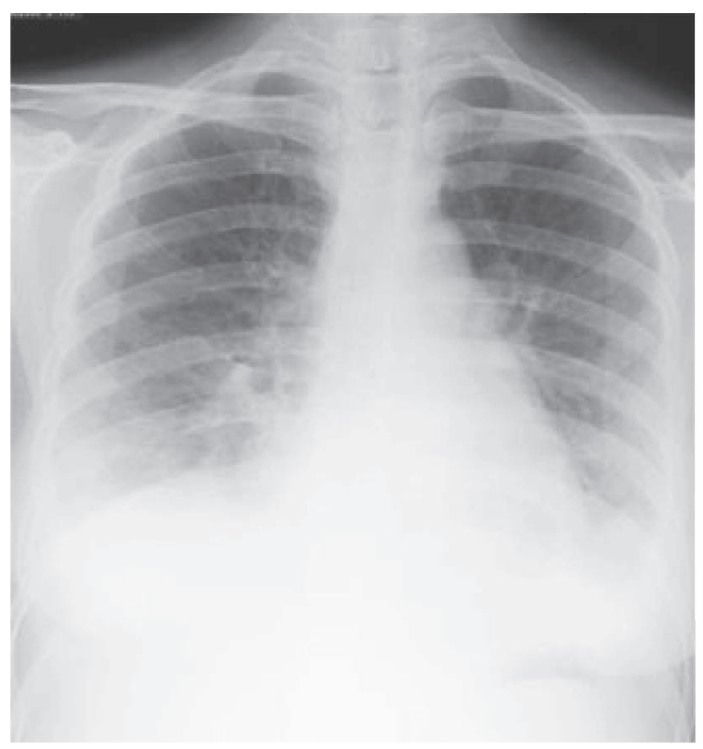

Day 0
B

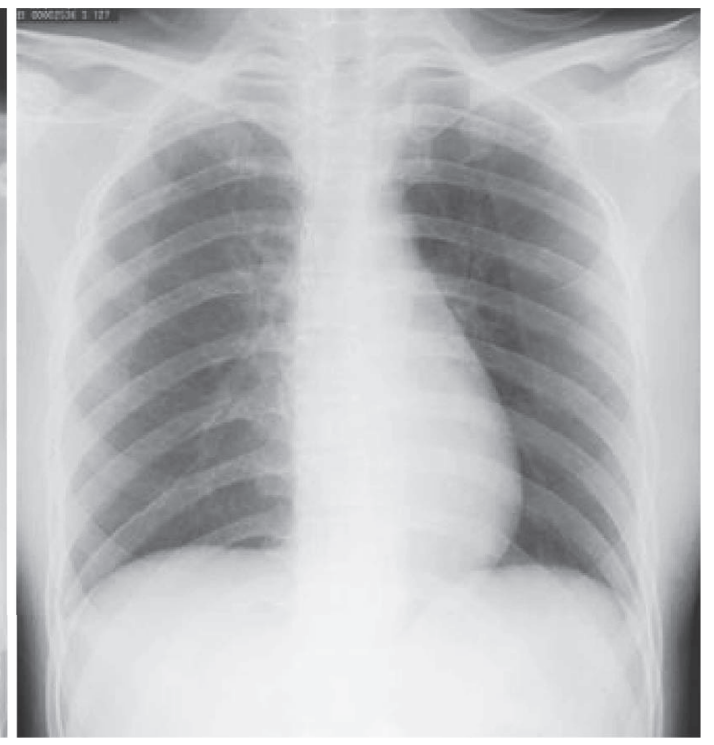

Day 3

Figure 1. Chest X-ray taken on admission, showing bilateral pulmonary infiltrates and pleural effusions (A). Chest X-ray taken on day 3, showing no pleural effusion (B).

rate $150 / \mathrm{min}$ and body temperature $35.0^{\circ} \mathrm{C}$. Her neck veins had collapsed and there was generalized edema which was pitting on the legs. Respiratory examination revealed dullness on percussion and decreased breath sounds at lung bases. Cardiovascular and abdominal examination showed no particular abnormality. Chest X-ray confirmed bilateral pleural effusions (Fig. 1a). Electrocardiogram and echocardiogram were normal. Hemoglobin was $22.8 \mathrm{~g} / \mathrm{dL}$, hematocrit $64.1 \%$, total leukocyte count $20,450 / \mu \mathrm{L}$, platelet count $221,000 / \mu \mathrm{L}$, blood urea nitrogen $31 \mathrm{mg} / \mathrm{dL}$, serum creatinine $1.67 \mathrm{mg} / \mathrm{dL}$, serum sodium $138 \mathrm{mEq} / \mathrm{L}$, serum potassium $5.2 \mathrm{mEq} / \mathrm{L}$, total serum protein $5.7 \mathrm{~g} / \mathrm{dL}$, serum albumin 3.0 $\mathrm{g} / \mathrm{dL}$ and ALT/AST 48/10 IU/L. IgG, IgA and IgM were $845.1 \mathrm{mg} / \mathrm{dL}, 98.0 \mathrm{mg} / \mathrm{dL}$ and $112.0 \mathrm{mg} / \mathrm{dL}$, respectively. Plasma level of lactate was $15.0 \mathrm{mg} / \mathrm{dL}$, pyruvate $0.6 \mathrm{mg} / \mathrm{dL}$ and CPK $68 \mathrm{IU} / \mathrm{L}$. Arterial blood gas analysis showed the following values: $\mathrm{pH}$ 7.26, $\mathrm{PaO}_{2} 53 \mathrm{mmHg}, \mathrm{PaCO}_{2} 45$ $\mathrm{mmHg}$ and $\mathrm{HCO}_{3}-19.6 \mathrm{mEq} / \mathrm{L}$. She was diagnosed with severe erythrocytosis and therefore initially referred to a hematologist. She was admitted and received 6 liters of intravenous fluid in 24 hours (a mixture of normal saline, crystalloid and colloid solutions) to maintain a systolic BP of 90 $\mathrm{mmHg}$ and high flow oxygen $7 \mathrm{~L} / \mathrm{min}$ to maintain $\mathrm{SpO}_{2}$ more than $90 \%$. She only passed $30 \mathrm{~mL}$ of urine on the first day. On the second day, she continued to require inotropic support and oxygen to maintain her systolic blood pressure above $90 \mathrm{mmHg}$ and oxygen saturation, but her urine output improved to $600 \mathrm{~mL}$ and her oxygen requirement decreased to $3 \mathrm{~L} / \mathrm{min}$. Her urine examination revealed trace protein and no active sediment. Hemoglobin fell to $11.0 \mathrm{~g} / \mathrm{dL}$ but there was no evidence of any bleeding. On the third day her urine output rose further to $2 \mathrm{~L} /$ day and she was taken off oxygen and inotropes. Chest X-ray showed no pleural effusion (Fig. 1b), suggesting that massive fluid recruitment from tissues into circulation and massive diuresis occurs because capillary barrier function has been restored.

To establish a diagnosis, the patient was transferred to our division of internal medicine. Extensive testing for possible causes was carried out. No infectious cause was found in any of these episodes. Furthermore, there was never any fever or documented focus. A computed tomography of the chest and abdomen, and cardiac functional tests revealed no abnormalities after the attack. Monoclonal immunoglobulin G-lambda was detected in the patient's serum by immunoelectrophoresis, but Bence Jones protein was negative. A bone marrow aspiration showed no abnormality. Notably, the level of G-CSF had increased to $57 \mathrm{pg} / \mathrm{mL}$ (normal: $<39 \mathrm{pg}$ / $\mathrm{mL})$. Other laboratory investigations, including interleukin-2 (IL-2) $(0.7 \mathrm{U} / \mathrm{mL}$, normal: $<0.8 \mathrm{U} / \mathrm{mL})$, interleukin-6 (IL-6) (1.4 pg/mL, normal: $<4.0 \mathrm{pg} / \mathrm{mL}$ ), vascular endothelial growth factor (VEGF) $(<31 \mathrm{pg} / \mathrm{mL}$, normal: $<38 \mathrm{pg} / \mathrm{mL})$, autoantibodies, complement studies, and $\mathrm{C} 1$ esterase inhibitor measurement, were all normal on admission. This young woman had experienced three episodes of unexplained shock in the previous 6 months, accompanied by evidence of capillary leakage in the form of generalized edema and bilateral pleural effusion, and was thus diagnosed with idiopathic systemic capillary leak syndrome by the presence of the characteristic triad of hypotension, hemoconcentration and hypoalbuminemia with monoclonal gammopathy. She was started on prophylactic treatment with beta-2 agonists and theophylline in combination with steroid therapy (prednisolone, $30 \mathrm{mg} /$ day) because it was reported that macromolecule leakage in response to various stimuli, including 


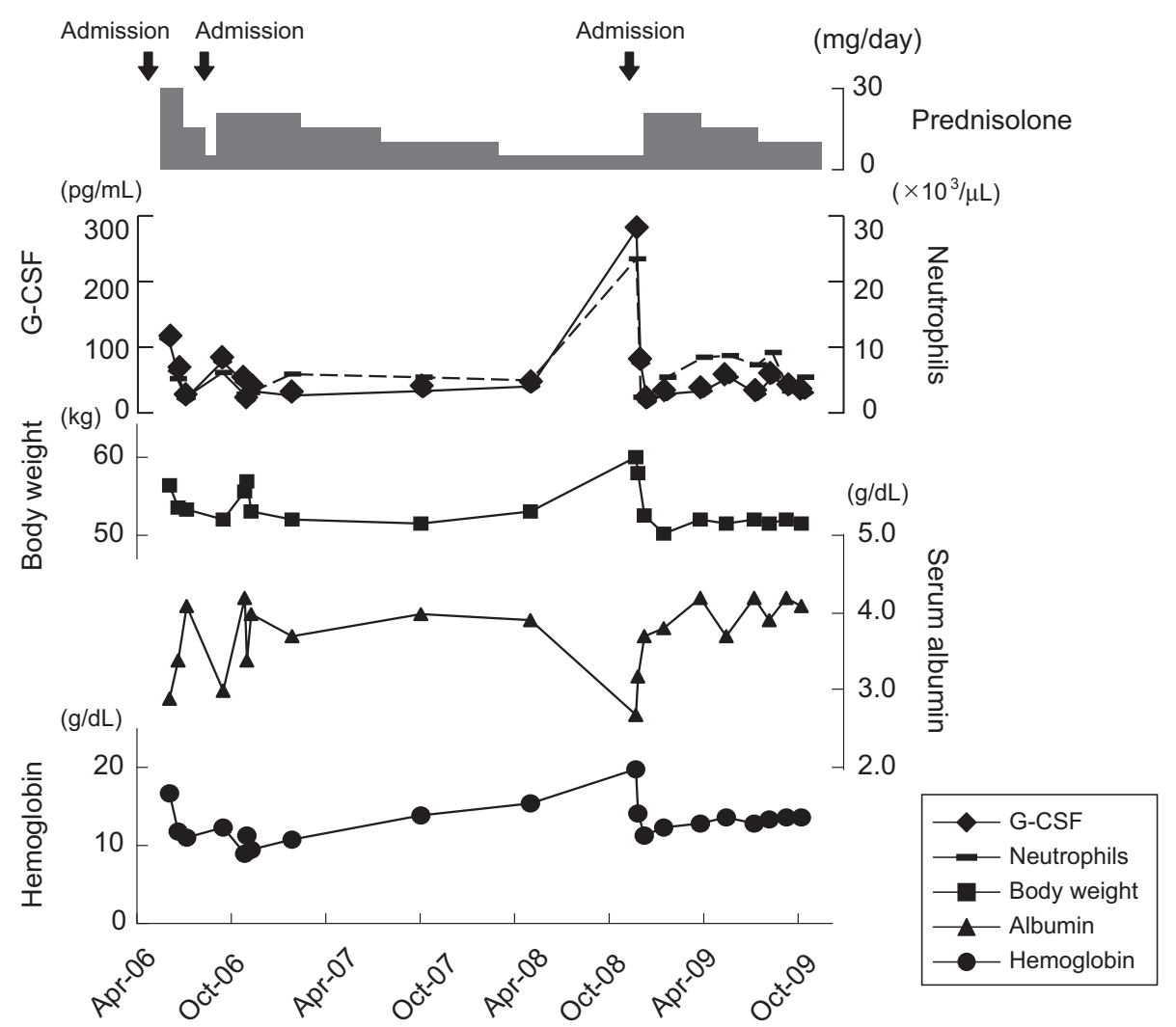

Figure 2. Clinical course of the present case. G-CSF: granulocyte colony-stimulating factor

histamine and bradykinin, can be inhibited by pretreatment with beta-2 agonists, theophylline and steroid therapy (2-4).

While tapering prednisolone, she was re-admitted in October 2006 and November 2008 with similar episodes of selflimiting shock, which were resolved within 4 to 6 days with supportive therapy in the form of intravenous fluids and inotropes. Again investigations failed to reveal any cause. Interestingly, the levels of serum G-CSF had increased (maximum, $279 \mathrm{pg} / \mathrm{mL}$ ) on admission and were significantly associated with the levels of blood neutrophils ( $\mathrm{r}=0.730, \mathrm{p}<$ 0.0001) (Fig. 2). After administration of prednisolone, GCSF levels showed a gradual decrease that coincided with improvement in the clinical course. Therefore, we used serum G-CSF levels as a marker of disease activity. Although she had chronic treatment with prednisolone ranging from $10 \mathrm{mg} /$ day to $20 \mathrm{mg} /$ day, she had self-limiting attacks, which were resolved within 3 days by drinking mineral water.

\section{Discussion}

To our knowledge, this is the first report in which G-CSF levels showed a relationship with the disease activity of SCLS attacks. Although the underlying pathophysiology is unknown, the presence of a monoclonal paraprotein is considered a characteristic manifestation of SCLS. In a review of the literature published in 2002 (4), 47 of 56 patients with SCLS had a monoclonal protein, predominantly IgGkappa. The cumulative probability of progression of mono- clonal gammopathy to myeloma or related plasma cell disorder has been reported as $10 \%$ at ten years, $21 \%$ at 20 years, and $26 \%$ at 25 years following diagnosis (7). There had been no reports of familial cases, until recently, a case of familial SCLS was reported (8). Despite many reports proposing mechanisms involving disparate biochemical substances or cells which might act on the capillaries to cause their leaking, no consistent mechanism has been proven (4-6). Serum levels of complements, kinins, prostaglandin, coagulation factors, histamine and serotonin are usually normal in patients with SCLS. Activation of the 5-lipoxygenase pathway and a role for IL-2, VEGF and interferon- $\beta$ has been suggested (9-12). It has also been reported that SCLS occurs after infusions of IL-2, IL-4, tumor necrosis factor, granulocyte-macrophage colony-stimulating factor (GM$\mathrm{CSF}$ ) and granulocyte colony-stimulating factor (G-CSF) as well as after autologous and allogeneic stem cell transplantation (13-15).

In the present patient, serum G-CSF levels, but not IL-2, IL-6 and VEGF levels, increased parallel to disease activity and were significantly associated with the levels of blood neutrophils. Recombinant human G-CSF increases neutrophil count and enhances their phagocytic and cytotoxic functions as well as their expression of adhesion molecules (16). Dagdemir et al reported that a patient developed SCLS twice while receiving G-CSF, without any time relationship between the onset of symptoms and the rapid increase in white blood cell count (17). They suggested that a direct effect of G-CSF on the endothelium may have played 
a major role and that G-CSF is able to cause SCLS even when the white blood cell count is very low. Based on these reports, G-CSF might have triggered neutrophil activation and the release of inflammatory mediators, resulting in tissue damage and systemic manifestations of increased capillary permeability in the present patient. It remains unknown what triggers and what kind of cells could release G-CSF during SCLS exacerbations. Bone marrow plasma cells might have the potential for release G-CSF because a monoclonal paraprotein presents in most patients with SCLS. We did not find a significant relationship between prodromal symptoms, such as flu-like symptoms and increases of serum G-CSF. Therefore, further investigation is needed to clarify the relationship between G-CSF and SCLS.

Treatment of SCLS with corticosteroids may be beneficial, since it interferes with granulocyte function and cytokine release (18). In fact, administration of prednisolone was effective in decreasing G-CSF levels with improvement in the clinical course in the present case. Furthermore, compounds that prevent cyclic adenosine monophosphate degradation and increase intracellular cyclic adenosine monophosphate levels, such as beta-adrenergic agonists and phosphodiesterase inhibitors (theophylline), have been used and are effective in many patients with SCLS (2-6). We therefore used beta- 2 agonists and theophylline for prophylactic treatment. In the present case, serum G-CSF levels were elevated before the treatment with prednisolone, theophylline, and terbutaline and showed a relationship with the clinical course; however, SCLS might not be due to a single pathogenesis. Nagao et al reported that 18 cytokines were measured and G-CSF, IL-6, IL- 8 and MCP-1 were significantly elevated (19), but we did not measure IL-8 and MCP-1. Therefore, in some SCLS, G-CSF might be useful, but not all cases. In the future, it is hoped that this avenue of research will clarify the pathophysiology and the treatment of SCLS.

In conclusion, although the pathophysiology of SCLS is unknown, we present a patient with SCLS who was treated successfully with corticosteroid, beta-2 agonists and theophylline and we found a relationship between the serum levels of granulocyte colony-stimulating factor and the clinical course. Thus, G-CSF might play an important role and can be a useful biomarker for the severity of attacks in SCLS.

The authors state that they have no Conflict of Interest (COI).

\section{References}

1. Clarkson B, Thompson D, Horwith M, Luckey EH. Cyclical edema and shock due to increased capillary permeability. Am J Med 29: 193-216, 1960.
2. Amoura Z, Papo T, Ninet J, et al. Systemic capillary leak syndrome: Report on 13 patients with special focus on course and treatment. Am J Med 103: 514-519, 1997.

3. Airaghi L, Montori D, Santambrogio L, Miadonna A, Tedeschi A. Chronic systemic capillary leak syndrome. Report of a case and review of the literature. J Intern Med 247: 731-735, 2000.

4. Kawabe S, Saeki K, Yamazaki H, Nagai M, Aoyagi R, Miyamura S. Systemic capillary leak syndrome. Intern Med 41: 211-215, 2002.

5. Dhir V, Arya V, Malav IC, Suryanarayanan BS, Gupta R, Dey AB. Idiopathic systemic capillary leak syndrome (SCLS): case report and systematic review of cases reported in the last 16 years. Intern Med 46: 899-904, 2007.

6. Druey KM, Greipp PR. Narrative review: The systemic capillary leak syndrome. Ann Intern Med 153: 90-98, 2010.

7. Kyle RA, Therneau TM, Rajkumar SV, et al. A long-term study of prognosis in monoclonal gammopathy of undetermined significance. N Engl J Med 346: 564-569, 2002.

8. Sion-Sarid R, Lerman-Sagie T, Blumkin L, Ben-Ami D, Cohen I, Houri S. Neurologic involvement in a child with systemic capillary leak syndrome. Pediatrics 125: e687-e692, 2010.

9. Rondeau E, Sraer J, Bens M, Doleris LM, Lacave R, Sraer JD. 5lipoxygenase pathway metabolites by peripheral leukocytes in capillary leak syndrome (Clarkson disease). Eur J Clin Invest 17: 5357, 1987.

10. Funke I, Prummer O, Schrezenmeier $\mathrm{H}$, et al. Capillary leak syndrome-associated with elevated IL-2 serum levels after allogeneic bone-marrow transplantation. Ann Hematol 68: 49-52, 1994.

11. Schmidt S, Hertfelder HJ, von Spiegel $T$, et al. Lethal capillary leak syndrome after a single administration of interferon beta- $1 \mathrm{~b}$. Neurology 53: 220-222, 1999.

12. Kinoshita Y, Kasaoka S, Fujita M, et al. Synchronized changes in serum vascular endothelial growth factor during the clinical course of chronic systemic capillary leak syndrome. Intern Med 49: 791794, 2010.

13. Orucevic A, Lala PK. Role of nitric oxide in IL-2 therapy-induced capillary leak syndrome. Cancer Metastasis Rev 17: 127-142, 1998.

14. Akasheh M, Eastwood D, Vesole DH. Engraftment syndrome after autologous hematopoietic stem cell transplant supported by granulocyte-colony-stimulating factor (G-CSF) versus granulocytemacrophage colony-stimulating factor (GM-CSF). Bone Marrow Transplant 31: 113-116, 2003.

15. Deeren DH, Zachee P, Malbrain M. Granulocyte colonystimulating factor-induced capillary leak syndrome confirmed by extravascular lung water measurements. Ann Hematol 84: 89-94, 2005.

16. Carulli G. Effects of recombinant human granulocyte colonystimulating factor administration on neutrophil phenotype and functions. Haematologica 82: 606-616, 1997.

17. Dagdemir A, Albayrak D, Dilber C, Totan M. G-CSF related capillary leak syndrome in a child with leukemia. Leuk Lymphoma 42: 1445-1447, 2001.

18. Mossad S, Kalaycio M, Sobecks R, et al. Steroids prevent engraftment syndrome after autologous hematopoietic stem cell transplantation without increasing the risk of infection. Bone Marrow Transplant 35: 375-381, 2005.

19. Nagao Y, Harada H, Yamanaka H, Fukuda K. Possible mediators for systemic capillary leak syndrome. Am J Med 124: e7-e9, 2011.

(C) 2011 The Japanese Society of Internal Medicine http://www.naika.or.jp/imindex.html 